\title{
Heavy ion collider project NICA/MPD at JINR (Dubna)
}

\author{
Grigory Trubnikov ${ }^{1}$, Alexander Kovalenko, Vladimir Kekelidze, Igor Meshkov, \\ Richard Lednicky, Alexei Sissakian, Alexander Sorin \\ JINR, Joliot Curie, 6, Dubna, Russia, 141980, E-mail: trubnikov@jinr.ru
}

\begin{abstract}
New project NICA/MPD (Nuclotron-based Ion Collider fAcility + MultiPurpose Detector) is under active development now at JINR (Dubna). The general goal of the project is to start in the coming 5 years an experimental study of hot and dense strongly interacting matter and search for possible signs of the mixed phase and critical endpoint in heavy ion collisions. This study should be carried out at nucleon-nucleon centre-of-mass energy ${\sqrt{\mathrm{S}_{\mathrm{NN}}}}=4-11 \mathrm{GeV}$ and average luminosity of $\mathrm{L}=10 \mathrm{E} 27 \mathrm{~cm}^{-2} \mathrm{~s}^{-1}$ for $\mathrm{Au}(79+)$. Another goal of the project is aimed to study spin physics with colliding beams of polarized protons and deuterons. The report contains physics motivation and main characteristics of the facility: scheme and operation scenario, proposed methods of intense ion beam formation and achievement of the required luminosity, conceptual design of the MPD. Status and plans of the project development are presented as well.
\end{abstract}

35th International Conference of High Energy Physics (ICHEP2010)

Paris,France

July 22-28,

$1 \quad$ Speaker 


\section{Introduction}

The flagship project of the Joint Institute for Nuclear Research (JINR) in Dubna in hign energy physics is now the NICA/MPD [1,2] started in 2007. This project is based on the existing superconducting (SC) synchrotron Nuclotron which is in operation since 1993 [3] and was modernized recently [4].

The first and main scientific goal of the NICA/MPD project is to search for new phenomena in heavy ion collisions such as the formation of a new form of matter - the compressed baryonic matter. This matter exists in neutron stars and in the cores of supernova explosions, while in the early Universe one meets the opposite conditions of very high temperature and a vanishing baryonic density (or baryonic chemical potential $\mu_{\mathrm{B}}$ ). In terrestrial experiments, high-density nuclear matter can be (transiently) created in a certain reaction volume in collisions of relativistic heavy ions. In these collisions a large fraction of the beam energy goes for hadron production, including excited resonances whose properties may be noticeably modified by the surrounding hot and dense medium. At very high temperatures or densities, the hadron mixture melts and its constituents form a new phase of matter - quarkgluon medium. The highest baryonic densities of the formed nuclear matter system can be achieved at Ö $_{\mathrm{NN}} \sim 4 \div 11 \mathrm{GeV} / \mathrm{u}$. That dictates a choice of colliding nuclei kinetic energy in NICA: $1 \div 4,5 \mathrm{GeV} / \mathrm{u}$. The same simulations show the colliding nuclei have to be neutron rich, as much as possible. Therefore gold ion beams are considered as the main candidates for this study. It is proposed that along with heavy ions NICA will provide polarized protons and deuterons.

\section{NICA operation}

Collider will be operated at a fixed energy without acceleration of an injected beam. Correspondingly the maximum energy of the experiment is determined by the Nuclotron magnetic rigidity that is equal to about $45 \mathrm{~T} \cdot \mathrm{m}$ at the field value of $2 \mathrm{~T}$. The collider rings will be placed one above the other, and the elements of SC magnetic system are being designed as a "twin bore" magnets. Circumference of the collider is close to $500 \mathrm{~m}$. For luminosity preservation in the heavy ion collision mode an electron and stochastic cooling systems are foreseen. To cover the total ion energy range the electron energy of the electron cooling system has to be varied from 0.5 to $2.4 \mathrm{MeV}$. For optimum operation of the stochastic cooling system the collider optic structure is designed to permit variation of the ring critical energy [5]. Collider optics is optimized to have maximal luminosity $\left(5 \times 10^{27} \mathrm{~cm}^{-2} \mathrm{~s}^{-1}\right)$ at the high energies $(4,5 \mathrm{AGeV})$. In the low energy range $(1,5 \mathrm{AGeV})$ luminosity is expected to be at the level of $5 \times 10^{25} \mathrm{~cm}^{-2} \mathrm{~s}^{-1}$. The future NICA facility will include: two injection chains, a booster synchrotron, the Nuclotron, collider rings, extracted beam channels from booster and Nuclotron (Fig.1),

To achieve the maximum design energy the Nuclotron has to accelerate fully stripped ions. To provide the ion stripping with a high efficiency the ions have to be accelerated to the energy of a few hundreds of $\mathrm{MeV} / \mathrm{amu}$. To reach this goal the superconducting booster synchrotron of 200 meters circumference will be build and put in operation.

The heavy ion beam accumulation in the collider rings will be realized with the help of the RF barrier bucket technique. The intensity of the injected portion influences the stacking 
process duration only and could be arbitrary in principle. The required beam emittance is formed during the stacking by the cooling application. The maximum bunch number in the collision mode is limited by the requirement to avoid parasitic collisions in the interaction region. The collider will be equipped with the Barrier Bucket RF system and two sinusoidal RF systems. One of them will be operated at the harmonic number coinciding with the bunch number at the collisione. Another one will be operated at significantly larger harmonic number that is necessary to keep a short bunch length at a reasonable RF voltage value.

The suggested project allows one to collide mass asymmetric beams including proton-ion (pA) collisions. Alongside of proper physics meaning, this is quite important as a reference point for comparison with the heavy ion data.
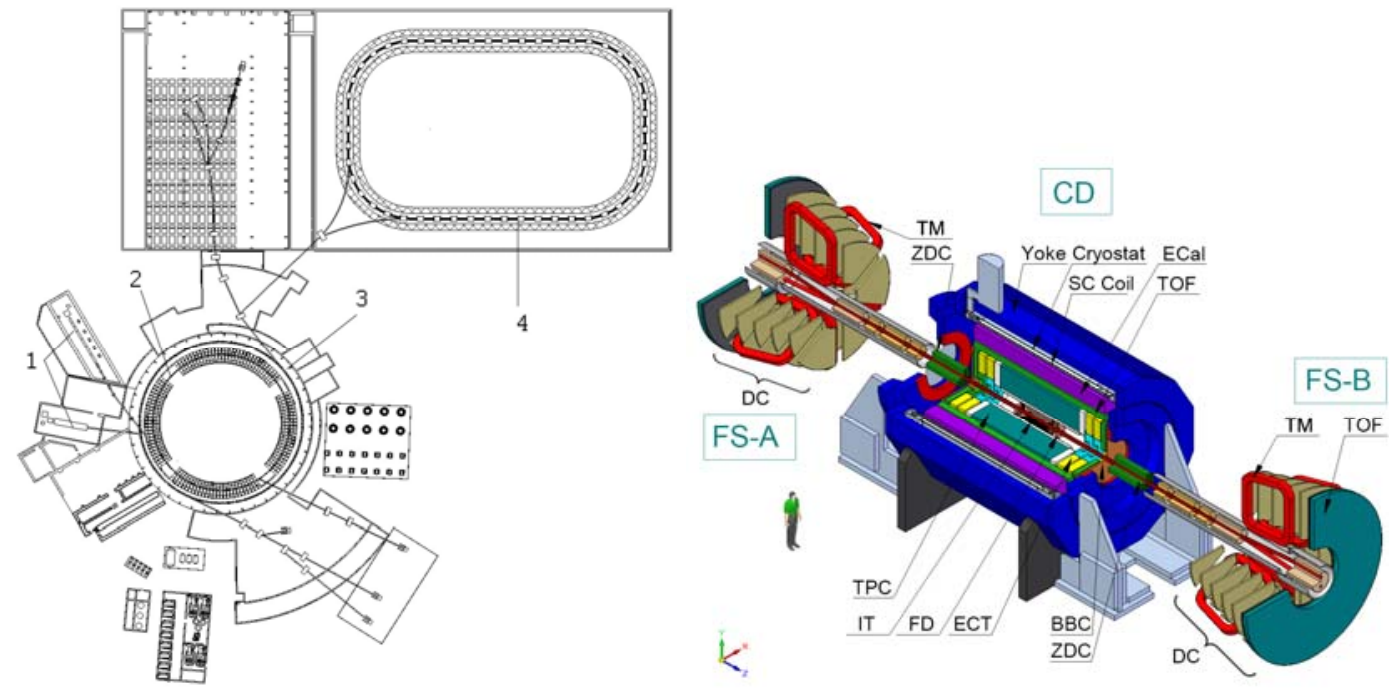

Fig. 1. Schematic view of the NICA accelerator complex (on the left): 1 - injector chains; 2 - Booster synchrotron; 3 - existing superconducting accelerator Nuclotron; 4 -Collider with two superconducting rings. Intersection points will be located at both long straight sections of the collider. Layout of the MPD detector (on the right).

Since the pA experiments will be performed with the same MPD detector the luminosity significantly larger than $10^{27} \mathrm{~cm}^{-2} \mathrm{~s}^{-1}$ is not necessary. This level is achievable quite easily because of large proton number in the beam comparing with heavy ions.

In this mode the collider injection chain has to be switched fast (during a time of a few seconds) from acceleration of heavy ions to acceleration of protons. Two acceleration and stacking chains of heavy ions and protons (or light polarized ions) are proposed:

- ESIS $\rightarrow$ HILAc $\rightarrow$ Booster $\rightarrow$ Nuclotron $\rightarrow$ Collider

- Duoplasmatron (polarized ions source) $\rightarrow$ LU-20 $\rightarrow$ Nuclotron $\rightarrow$ Collider

The proton beam generated by the duoplasmatron source is accelerated by LU-20 up to energy of $20 \mathrm{MeV}$. Single turn injection allows Nuclotron (without booster) to have more than $10^{11}$ protons. Another mode of the facility operation will be proton-proton and deuterondeuteron polarized beam collisions in the energy range $5 \div 12.6 \mathrm{GeV}$ for protons and $2 \div 5.8$ $\mathrm{GeV} / \mathrm{amu}$, for deuterons, respectively. The luminosity above $1 \cdot 10^{31} \mathrm{~cm}^{-2} \mathrm{~s}^{-1}$ is required over the total energy range.

In the Nuclotron ring deuteron depolarization resonances are absent in the whole achievable energy range. The possibility of acceleration and extraction of polarized deuterons in the Nuclotron has been demonstrated a few years ago. The measurements of the polarization 
degree on internal and extracted beams performed by three independent groups gave the value of $65 \%$ in agreement with the expected value. For acceleration of the polarized proton beam the Nuclotron has to be equipped with insertion devices for the spin tune control to cross the depolarization resonances without loss of the polarization degree.

The collider operational cycle assumes feeding the collider with ions during a few minutes after that the collision experiment will be provided during a few hours at almost constant luminosity without additional injections. At this time the Booster and Nuclotron will be used for independent experimental programs. The Nuclotron with LU-20 as injector will provide light ion beams for internal target experiments, and its slow extraction system will be used for fixed target experiments and tests of the MPD elements. The Booster will be used as a heavy ion synchrotron. Its designed magnetic rigidity of $25 \mathrm{~T} \cdot \mathrm{m}$ allows for a wide range of radiobiological and applied experiments as well as cancer therapy research with carbon and other ions [6].

\section{The MPD apparatus}

The major physics points that determine the conceptual design of MPD are as follows: 1) to probe the deconfinement phase transition; new very precise measurements of hadron yields including multi-strange baryons; 2) a measurement of fluctuations and correlation patterns in the vicinity of the QCD Critical End Point, which requires an apparatus with a solid angle coverage close to $4 \pi$ and excellent particle identification capability; 3) to study in-medium modifications of hadron properties; the invariant mass spectra of dielectrons should be measured up to $\mathrm{M}\left(\mathrm{e}^{+} \mathrm{e}^{-}\right) \approx 1 \mathrm{GeV} / \mathrm{c}^{2}$ in a variety of colliding systems; 4) finally, a detector to study heavy-ion collisions in a high track multiplicity environment has to be functional at high interaction rates; fine granularity of detector elements and good event characterization capability are also required.

The MPD is designed as a $4 \pi$ spectrometer capable of detecting charged hadrons, electrons and photons in heavy-ion collisions in the energy range of the NICA collider. To reach this goal, the detector will include a precise 3-D tracking system and a high-performance particle identification system based on time-of-flight measurements and calorimetry. At the design luminosity, the event rate in the MPD interaction region is about $7 \mathrm{kHz}$; the total charged particle multiplicity exceeds 1000 in the most central $\mathrm{Au}+\mathrm{Au}$ collisions at ${\sqrt{\mathrm{S}_{\mathrm{NN}}}}=11 \mathrm{GeV}$. As the average transverse momentum of the particles produced in a collision at NICA energies is below $500 \mathrm{MeV} / \mathrm{c}$, the detector design requires a very low material budget. The general layout of the MPD apparatus is shown in Fig.1. The detector setup includes the Central Detector (CD) covering \pm 2 units in pseudorapidity $(\eta)$ and two forward spectrometers (FS-A,B) for $2<|\eta|<3$ (optional). The whole $\mathrm{CD}$ will be a $6.5 \mathrm{~m}$ long cylinder of about $5 \mathrm{~m}$ in diameter. These design parameters are determined by several technical constraints and guided by a trade-off of efficient tracking and PID against a reasonable material budget. A more detailed description of the detector components can be found in ref. [7]. 


\section{Plans for realization}

The Nuclotron upgrade program considered as a first stage of the NICA project [4] is in the final stage now. The main goal of the program is to prepare the synchrotron for operation as a part of the NICA collider injection chain. To the moment the upgrade of the Nuclotron vacuum system is completed, deep reconstruction of liquid helium production facility was provided during 2008-2009. The modernization of the magnetic system power supply and energy evacuation system will be completed this year. As a result of the works at the Nuclotron run in March 2010 the ions $\mathrm{Xe}^{42+}$ were successfully accelerated up to energy of about 1.5 $\mathrm{GeV} / \mathrm{amu}$, and the magnetic system was operated at the dipole magnetic field of about $1.8 \mathrm{~T}$ (close to the designed value of 2T). Development of the new heavy ion source and the polarized ion source is the part of the Nuclotron upgrade as well. A project of the infrastructure reconstruction is under development by the State Specialized Design Institution (Moscow) and it's completion is expected at the beginning of 2011; as a result the expenses and the required reconstruction period will be determined.

The structural dipole and quadrupole magnets for the collider, as well as for the Booster, will be based on the design developed during the Nuclotron construction [8,9]. The Nuclotron magnet fabrication has brought a great experience to the Institute staff in the field of SC magnet design and manufacturing. Such types of magnets are planned to use for construction of SIS-100 synchrotron in the FAIR project. The construction of magnet prototype based on the preliminary design has been started in 2010 .

To construct the Booster and collider rings it is planned to fabricate more than two hundreds of dipole magnets and lenses during short period of time. The working area for the magnet fabrication and test benches required for the magnet commissioning are under preparation now.

In the optimistic expectations the experiments with circulating beam in the collider rings can be started at the end of 2015. At the first stage of the collider operation the heavy ion collisions will be realized and the design luminosity level can be achieved by 2017. After upgrade of ring optics in the vicinity of collision point the heavy ion-proton collisions will be performed. The collisions of light polarized ions are scheduled for the third stage of collider operation.

\section{References}

[1] A. N. Sissakian et al., "The Project NICA/MPD at JINR: Search for the mixed phase of strongly interacting matter at Nuclotron-based ion collider facility" XXIII Int. Symposium on lepton and photon interaction at high energy, LP07, Daegu, Korea, 2007.

[2] Nuclotron-based ion collider facility, http://nica.jinr.ru/

[3] A.D.Kovalenko., "Status of the Nuclotront", EPAC'94, London, June 1994, Proc. of v.1, pp. 161-164, (1995).

[4] A. Butenko, G. Trubnikov et al., Status of the "Nuclotron-M" Project, Proc. of IPAC'10, Kyoto, Japan, 2010, p.684

[5] Kostromin, O. Kozlov et al., Lattice of the NICA Collider Rings, Proc. of IPAC'10, Kyoto, Japan, 2010, p.6907

[6] I. Meshkov, A. Sisakian, G. Trubnikov, Heavy Ion Programs for Applications and Fundamental Research at JINR, Proc. of IPAC'10, Kyoto, Japan, 2010, p.1319.

[7] V.D.Kekelidze, A.S.Sorin et al.,The MultiPurpose Detector (MPD). Conceptual Design Report, v1.0, http://nica.jinr.ru/

[8] A. Butenko at al., "Design of the Nuclotron booster in the NICA project", Proc. of IPAC'10, Kyoto, Japan, 2010, pp. 681.

[9] H. Khodzhibagiyan, N. Agapov, A. Kovalenko, A. Smirnov, A. Starikov "Development of fast-cycling superconducting magnets at JINR", Proc. of the Twenty First Int. Cryogen. Eng. Conf., ICIC21, CRYOPrague 06, vol.1, Prague, Czech Republic, 2006, pp. 113-116. 